\section{Response to both}

When developing our review we were keen to generate discussion with a view to raising awareness of, and improving practice around, discharge planning. We therefore appreciate the comments of Drs Levine and Leung.

We agree that sharing accurate information in a timely manner is a key factor in both discharge planning and provision of high-quality care. The presence on ward rounds of nursing staff should improve this, but can be suboptimal. Combined paperwork and single assessment pathways, alongside daily targeted multidisciplinary meetings have been used in the admission unit at Stafford with some effect. Many other assessment units (eg Wolverhampton) provide active elderly care in-reach services and/or regular consultant input, potentially facilitating more appropriate discharge and admission.

Admissions units are a hub in most acute hospitals, and accurate assessment and decision making, with early senior clinician involvement at the point of admission, can ultimately improve care downstream. We would endorse the points raised by Dr Leung regarding the potential conflict between early discharge and the provision of high-quality care, and the fact that many frail patients with complex medical conditions and social circumstances cannot be discharged directly from admissions units.

Pathways/protocols for early discharge of patients with selected conditions (eg deep vein thrombosis, cellulitis) from admission units have been described. ${ }^{1,2}$ We also recognise concerns of colleagues around setting discharge dates, and use the term 'provisional discharge date' in the notes, thus allowing flexibility.

Comprehensive assessment for many patients requires admission to specialist elderly care wards, where time will allow multidisciplinary assessment to occur. Our review aims to improve discharge planning both from admissions areas and specialist wards, improving the flow of patients through hospital, to allow more efficient use of resources.
Regular formal multidisciplinary meetings that document clear plans, proposed timescales, and individual responsibilities (either in clinical notes or on multidisciplinary handover sheets) can advance this process. We have also found that whiteboards are helpful in focusing actions of members of the multidisciplinary team.

Discharging patients, both from admissions unit and elderly care wards, can be a challenging process, and should be actively taught to doctors in training. Unsafe discharges reflect poor care and are unacceptable.

PHILIP DAINTY

Locum consultant physician Emergency Assessment Unit New Cross Hospital, Wolverhampton JIMMY ELIZABETH

Consultant physician Stafford General Hospital, Stafford

\section{References}

1 Boccalon $\mathrm{H}$, Elias A, Chale JJ et al. Clinical outcome and cost of hospital vs home treatment of proximal deep vein thrombosis with a low-molecular-weight heparin. Arch Intern Med 2000;160: 1769-73.

2 Corwin P, Toop L, McGeoch G et al. Randomised controlled trial of intravenous antibiotic treatment for cellulitis at home compared with hospital. BMJ 2005; 330:29-135.

\section{A postal survey of doctor's attitudes to becoming mentally ill}

Editor - I read with great interest Hassan et als excellent paper (Clin Med August 2009 pp 327-32). There is much research and publicity, for example a recent Department of Health review, ${ }^{1}$ which finds that there are higher rates of psychological illnesses in doctors than the general population, so it is surprising to find in this study that the majority of doctors, especially psychiatrists, were not aware of this fact. Overall it seems to me that as a profession we are having the same 'conversation' about this issue. The debate on how to provide services for sick doctors has continued in a similar way for years. One of the factors that bothers doctorpatients most, indeed many patients, is the stigma of illness and sickness absence, as is borne out in this study. It is interesting that in reality most doctors do not lose their jobs, or have any long-term problem resulting from the fact of a particular diagnosis or sickness absence. It is true that long-term sickness absence requires a multidisciplinary approach and that the earlier a problem is dealt with the better. Many of a doctor's problems with illness arise from a denial of them. Confidentiality, which is different, and as borne out in this study, is the key to managing these situations. Many services for doctors do exist, can be accessed via their general practitioners or occupational health departments, and can be off-site from the workplaces.

I am surprised that most respondents say they would talk to family and friends because in many cases, in my experience as an occupational physician, once doctors and other people are ill, they find that admitting illness to family is difficult. Those answers smack of denial as well and may be a source of information bias in the study.

Most interesting is that the authors say that these attitudes and perceptions about doctors' health should be tackled and changed at medical school level as I am currently carrying out this kind of work with students. I have found, sadly, that the attitudes written about in this paper are well-entrenched by mid-third-year stage.

CLARE RAYNER

Specialist occupational physician Trainer for National Education Programme

for Health and Work

Royal College of General Practitioners

\section{Reference}

1 Department of Health. Mental health and ill health in doctors. London: DH, 2008.

\section{The skin in general medicine}

Editor - Dhoat and Rustin reviewed an important but often neglected part of general medicine in their article (Clin Med 